\begin{tabular}{|c|c|c|}
\hline Beitr. Ent. & Keltern & ISSN 0005 - 805X \\
\hline $\mathbf{5 6}(2006) 2$ & S. 406-421 & 15.12 .2006 \\
\hline
\end{tabular}

\title{
Zur Kenntnis der Collembolen in der Paläarktis
}

\section{(Insecta, Entognatha)}

Mit 5 Figuren und 4 Tabellen

\section{WOLFram DUNGer}

\section{Zusammenfassung}

Für die Springschwänze (Collembola, Insecta) gibt es weder historisch noch aktuell einen Überblick über die Fauna der Paläarktis. Die taxonomische und faunistische Forschung erbrachte in den letzten Jahrzehnten einen sprunghaften Anstieg der Kenntnisse sowohl im europäischen als auch im asiatischen Teil. Seit 1994 gibt der Autor am Staatlichen Museum für Naturkunde Görlitz die „Synopses on Palaearctic Collembola“ als Vorläufer einer späteren Paläarktis-Fauna heraus. Die bislang erschienenen 4 Bände (Hypogastruridae, Tullbergiinae, Isotomidae, Symphypleona) dokumentieren, dass 57 \% der Arten dieser Taxa später als 1960 beschrieben wurden und 41 \% ihren Schwerpunkt im asiatischen Teil der Paläarktis haben. Gegenwärtig ist die Gesamtzahl der paläarktischen Arten auf 3100 zu schätzen. Die Arbeit informiert über Probleme der Taxonomie der Collembola und präsentiert den Editionsplan der „Synopses“ bis 2010.

\section{Summary}

A survey of Palaearctic Collembola has never been published so far. Taxonomical and faunistical papers of the last decades brought about a sharp increase of the knowledge for the European and Asiatic regions as well. Since 1994, the author edites the "Synopses on Palaearctic Collembola" at the State Museum of Natural History Görlitz as a precursor of a Palaearctic Fauna. So far, four volumes appeared (Hypogastruridae, Tullbergiinae, Isotomidae, Symphypleona). $57 \%$ of the species of these supertaxa were described later than 1960 , and $47 \%$ are mainly distributed in the asiatic part of Palaearctis. The total number of Palaearctic species can be estimated as 3100. The paper informs about problems in Collembolan taxonomy and shows the plan of edition of the "Synopses" up to 2010.

\section{Keywords}

Collembola, species numbers, taxonomy, Palaearctis

\section{Collembola: Charakteristik}

Collembolen sind bereits aus dem Unterdevon von Rhynie bekannt und somit die ältesten paläontologisch nachgewiesenen Insekten. Die Frage, ob sie überhaupt Insekten sind, unterliegt einer Diskussion, die gegenwärtig zur positiven Antwort neigt (BrTsCH et al. 2004, Giribet et al. 2004). 
Morphologisch fallen die Collembolen durch ihre primäre Flügellosigkeit, das nur 6gliedrige Abdomen und die charakteristische Umgestaltung der Abdominalbeine in den Ventraltubus, das Retinaculum und die Furca auf. Die Atmung läuft über die zarte Haut ab. Die Mundwerkzeuge sind in einer Hautfalte verborgen.

Ihre morphologische Ausstattung deutet auf eine primäre ökologische Anpassung an einen Wechsel zwischen kryptozoischer Lebensweise (Aufsuchen von Refugien gegen Austrocknung und Einstrahlung) und Weidegänger auf relativ offenem, von Algen und Thallophyten überzogenem, festen Substrat, wo sie eine Sprunggabel brauchten, um sich durch Fluchtsprünge vor Freßfeinden in Sicherheit zu bringen, und wo sie mit dem Ventraltubus Feuchtigkeit aufnehmen und sich notfalls anheften konnten (Dunger 1994, D’Haese 2002 ). Der Name Collembola verewigt einen Irrtum: er bezieht sich auf den Ventraltubus und bedeutet "Leimkolbenträger" (kolla = Leim, embolon = Kolben, Keil). Das Anheften beruht jedoch auf Ansaugen (ohne Leim).

Collembolen sind mit Individuendichten von 10000 bis 400000 pro m² Boden allgegenwärtig und damit wohl die individuenreichste Gruppe der Insekten.

Dass wir gegenwärtig nur magere 7000 Arten unterscheiden können (DEHARveng 2004), hängt wohl mit dem Nachhinken ihrer Erforschung hinter derjenigen der übrigen Insekten zusammen.

\section{Entwicklung der Kenntnis}

Schon Aristoteles hat Collembolen als rötliche und behaarte Schneeflöhe wahrgenommen, doch LinNé konnte 1758 noch immer nur 10 Collembolen-Arten aufzählen. Der eigentliche Entdecker und Namensgeber war Sir Joнn Lubвоск in London, der in seiner Monographie (1873) bereits 130 Arten vergleichsweise modern beschrieb. Um 1900 waren etwa 180 Arten aus Europa bekannt (RuseK 2002). Zwischen Lubbocks Monographie und der Weltliste des Australiers Salmon (1964) liegt die klassische Periode der Entwicklung der Collembolenkunde, also im wesentlichen im 20. Jahrhundert (Hopkin 1997).

Zentren der Erforschung waren zunächst das nördliche und westliche Europa, bald auch ausgedehnt auf das Mittelmeergebiet und Nordamerika. Die schnell anwachsende Kenntnis für Zentraleuropa fasste Handschin (1929) mit 212 Arten und sein Nachfolger Gisin in seiner Europafauna (1960) mit 867 Arten zusammen. Dem folgte die bedeutende Monographie von Sтасн (1949 - 1963) und die Bearbeitung der Fauna Mitteleuropas im „Brohmer“ durch Palissa (1964).

Diese Serie großer Faunenwerke im europäischen Bereich brach 1964 abrupt ab, aber die Zahl der Artbeschreibungen stieg weltweit, auch in Europa, rasant an (Fig. 1).

Diese Intensivierung der Collembolenforschung betraf in der zweiten Hälfte, besonders im letzten Viertel des 20. Jh., zunehmend auch den asiatischen Raum; begonnen in der japanischen Region durch YosII (etwa 50 Arbeiten 1939 - 1992), im asiatischen Teil Russlands durch Martynova (über 30 Arbeiten 1964 - 1986) und in der Mongolei durch Dunger (1978 - 1985) und Betsch (1977).

Ergänzt wurden diese Kenntnisse vor allem durch Übersichten für die Nearktis durch Christiansen und Bellinger (1980/1991) und für die Arktis und Nordeuropa durch 
FJellberg $(1985,1998)$. Für Russland, insbesondere die weiten asiatischen Gebiete, bildete sich die erfolgreiche, von Frau Prof. Chernova geleitete Moskauer Schule (Babenko et al. 1994). Dokumente des Fortschrittes auch in der Erforschung der europäischen Collembolenfauna sind die Checklists vieler Länder (z. B. DALlaI et al. 1995 für Italien, JorDANA et al. 1990 für die Iberische Halbinsel).

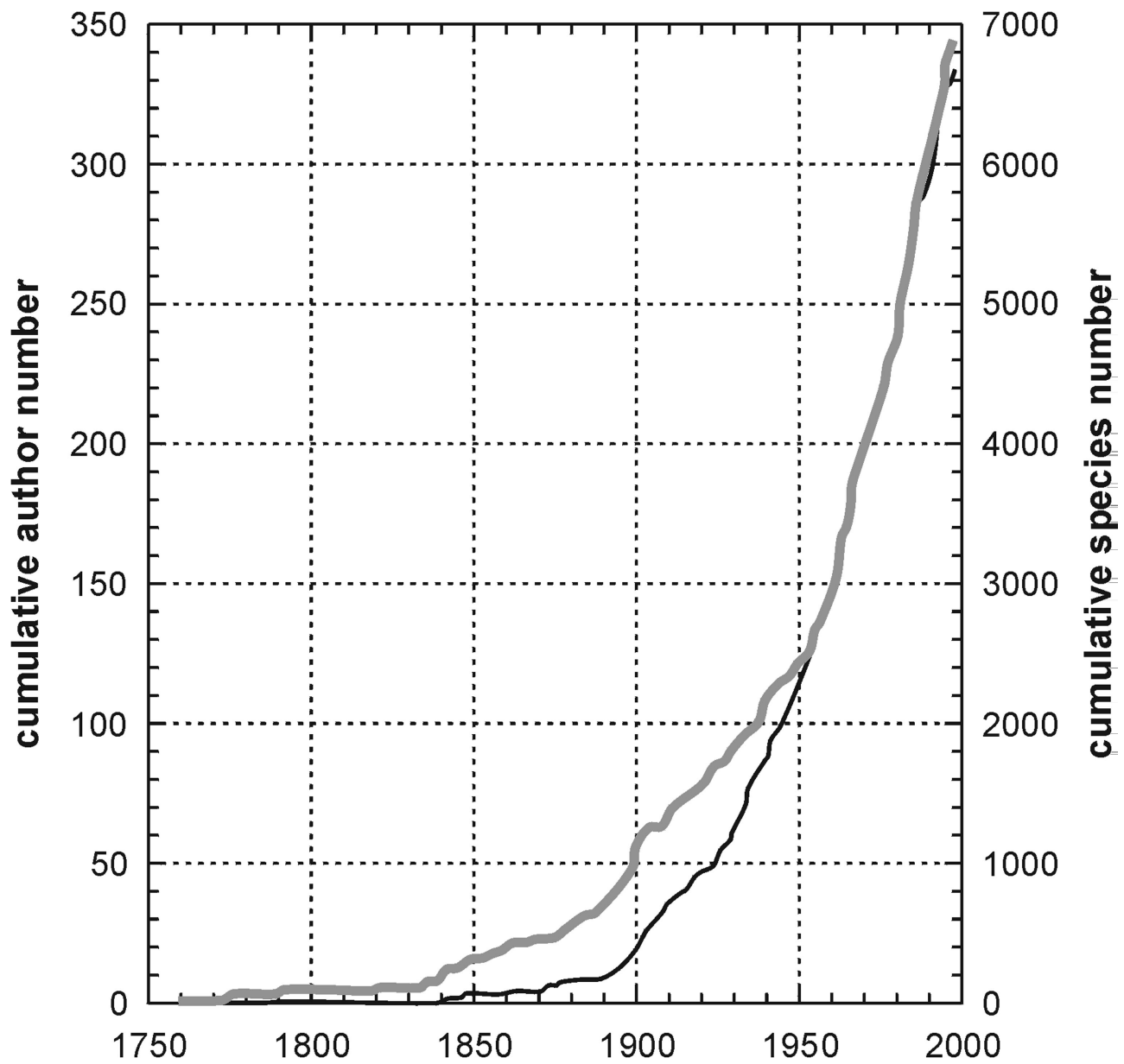

Fig. 1: Collembolen der Welt: Kumulative Kurven der bekannten Artenzahlen bis 2000 (dünne Linie) und Zahlen der an der Artbeschreibung beteiligten Autoren (dicke Linie). Nach DeHarveng (2004).

Ab etwa 1980 war die Bestimmung von Collembolen in Europa ohne Berücksichtigung der asiatischen Fauna nicht mehr sinnvoll und für Nicht-Spezialisten schon deshalb kaum möglich, weil die vorhandene Determinations-Literatur nur die Hälfte der beschriebenen europäischen und überhaupt keine asiatischen Arten führte.

Es wurde also Zeit, eine Übersicht über die Collembolenfauna der gesamten Paläarktis zu gewinnen. 
Paläarktis-Forschung

Umgrenzt ist die Paläarktis durch das Nordpolarmeer, den Pazifik und den Atlantik. Die südliche Begrenzung bildet in der Äthiopischen Region zunächst der nördliche Wendekreis; ab dem Golf von Oman folgt sie dem 30. Grad nördlicher Breite von der Indischen Region bis zum Chinesischen Meer südlich des Yantze Kiang River bzw. von Shanghai.

Korrekturen der Paläarktisgrenze haben einige Spezialisten nach der Bewertung für ihre Tiergruppe vorgenommen, so für das beliebte Exkursionsgebiet Nepal, das vorwiegend südlich des 30. Breitengrades liegt. Bretfeld (1999) klammert Nepal für die Kugelspringer als tropisch aus, ThiBaud et al. (2004) für die Hypogastruriden und Deharveng (2006) für die Neanuridae halten die nepalesische Fauna dagegen für deutlich paläarktisch.

Die Erforschung der Collembolenfauna großer Landteile Zentral-Asiens, des nördlichen Vorderindien und China, oder auch von Kamtschatka und Sachalin hat gerade erst begonnen. Von hier bis hinauf zur Behringstraße vermutet Cassagnau (1989) wichtige Ausbreitungszentren von heute weit verbreiteten Gruppen von Poduromorphen. Eine biogeographische Sonderstellung wird auch für die makaronesischen Inselgruppen angenommen (FJELlberg 1995).

Das Vorliegen der ersten vier Bände der Synopses der Paläarktis bietet erste Einblicke in das Verbreitungsverhalten der Arten (Tab. 1). So haben 52,7 \% der bisher für die Paläarktis erfassten Arten ihren Verbreitungsschwerpunkt in Europa, während 41,3 \% vorrangig asiatisch und 5,9\% vorrangig nearktisch verbreitet sind. Die biogeographische Orientierung an den für Collembolen relevanten geographischen Strukturen steht noch aus.

Tab. 1: Anzahl und Verbreitungsschwerpunkte der paläarktischen Collembolen-Arten von vier Supertaxa in Europa, Asien und der Nearktis nach dem Kenntnisstand der Bände I - IV der Synopses on Palaearctic Collembola (1994 - 2004).

\begin{tabular}{|l|l|l|l|l|}
\hline Supertaxon & Paläarktis & Europa & Asien & Nearktis \\
\hline $\begin{array}{l}\text { Hypogastruridae } \\
\%\end{array}$ & 330 & 187 & 118 & 25 \\
& 100 & 56.7 & 35.7 & 7.6 \\
\hline $\begin{array}{l}\text { Tullbergiidae } \\
\%\end{array}$ & 75 & 68 & 4 & 3 \\
\hline $\begin{array}{l}\text { Isotomidae } \\
\%\end{array}$ & 100 & 90.6 & 5.3 & 4.0 \\
\hline Symphypleona & 260 & 342 & 243 & 30 \\
$\%$ & 100 & 55.6 & 39.5 & 4.9 \\
\hline Total & 1280 & 38 & 164 & 18 \\
$\%$ & 100 & 575 & 63.1 & 6.9 \\
\hline
\end{tabular}

Wirklich rein europäische Verbreitungsareale sind besonders für einige große, oberflächenbewohnende, seltener auch für kleinere, bodenbewohnende Arten der Collembolen bekannt. Mit dem Anwachsen der Kenntnis mehrt sich nun die Zahl der Arten, für die sich europäisch-asiatische Mischareale ergeben. Als Beispiel sei BreTfELD (1989) zitiert, der für Heterosminthurus bilineatus eine rein europäische Verbreitung beschrieb. Fast 
gleichzeitig mit dem Erscheinen dieser Arbeit bekam er die ersten asiatischen Exemplare aus der Region Tuwa (unweit des Baikalsees) vorgelegt. Nur sein Urteil als Spezialist konnte sichern, dass sich hiermit tatsächlich neue Dimensionen der Verbreitung dieser (und nicht einer verwandten) Art eröffneten. Wir stehen am Anfang einer Progression des Wissens, die notwendig von taxonomischer Kenntnis getragen sein muss.

\section{Taxonomie der Collembola}

Ein aktueller Überblick mit dem Anspruch einer Synopsis der paläarktischen Fauna muss notwendigerweise alle vorhandenen Daten an der heutigen Kenntnis der Taxonomie messen. Damit wird die Taxonomie der Collembola zum kritischen Punkt der Erarbeitung dieses Werkes, das mindestens in diesem Bereich nicht nur kompilatorisch sein kann. Hier sollen nur die wichtigsten in diesem Bereich anstehenden Fragen kurz skizziert werden.

Chaetotaxie: Die bevorzugte Basis der Taxonomie ist heute die Chaetotaxie als nichtadaptives Merkmal. Sie beschreibt nach weltweit mühsam annähernd vereinheitlichten Mustern Lage und Zahl bestimmter Borstentypen längs des Körpers, vor allem Grundborsten, Makrochaeten und Sinnesborsten (Sensillen) (Fig. 2). Die Sensillenformel hat eine besondere Bedeutung erlangt, denn sie ist spezifisch für phyletische Linien.

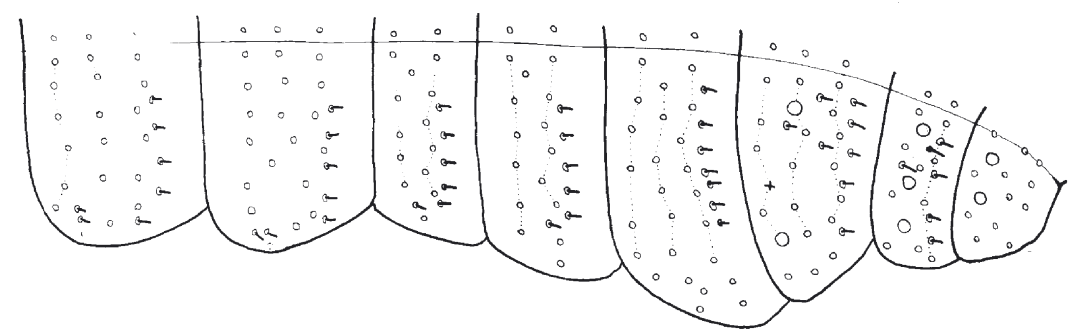

Fig. 2: Dorsale Chaetotaxie (linke Körperseite; Thorax II-III und Abdomen I, II, III und IV-VI) von Isotomurus palustris (Müller, 1776) im 1. Stadium. Die Insertionsstellen der Grundborsten sind durch kleine Ringe, die der Makroborsten durch große Ringe gekennzeichnet. Sensillen sind ausgezeichnet. Nach Deharveng (1979).

Leider ist das mikroskopische Erkennen der Sensillen wegen ihrer minimalen Größe und oft versteckten Lage sehr anspruchsvoll. Vor allem deshalb ist die Chaetotaxie bisher nur für $50-70 \%$ der Arten ausreichend beschrieben. Hohe Bedeutung hat die Juvenilchaetotaxie erlangt, die phyletische Verwandtschaft auf Gattungsebene erkennen lässt. Das Studium der ersten Juvenilstadien setzt zwar oft eine Zucht der Art voraus, dennoch beruht die moderne Taxonomie, z. B. der Onychiuridae, schon heute wesentlich hierauf.

Neue Merkmale: Die Ausbildung bislang wenig beachteter taxonomischer Merkmale, z. B. der Feinmorphologie von Mundteilen und Sinnesorganen, eröffnet neue Möglichkeiten der Charakterisierung. Auch diese liegen oft an der Grenze der Erkennbarkeit mit dem Lichtmikroskop; ihr Studium erfordert oft eine spezielle Präparation.

Untaugliche Merkmale: Die Überwindung alter Art-Merkmale betrifft vor allem die Notwendigkeit, die Beschreibung von Pigmentmustern in der Artdiagnose vorrangig epedaphischer Supertaxa durch konstante und prüfbare Merkmale zu ersetzen. 

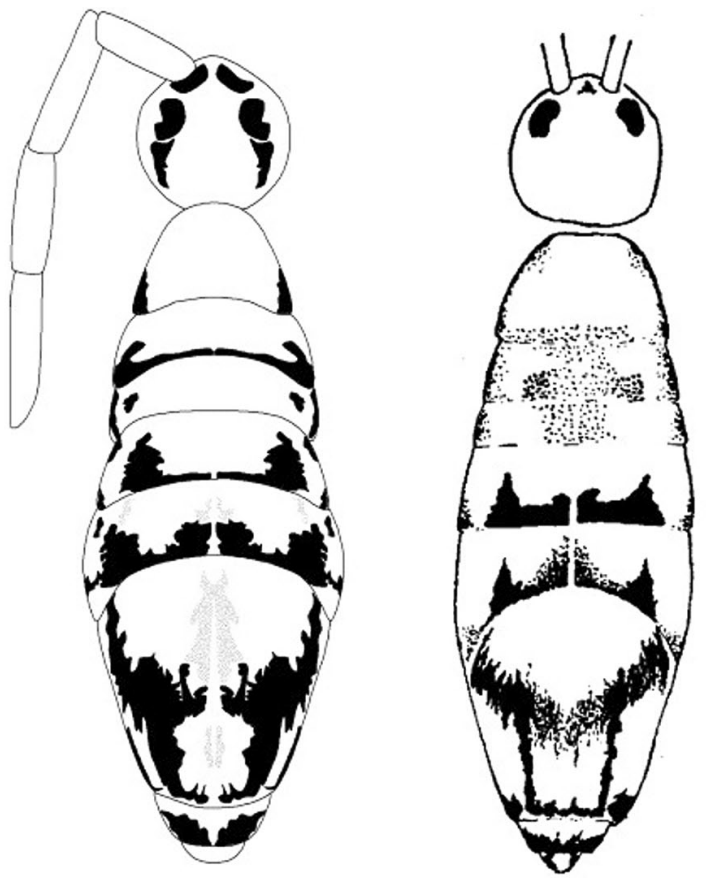

E. nivalis Linnaeus, 1758
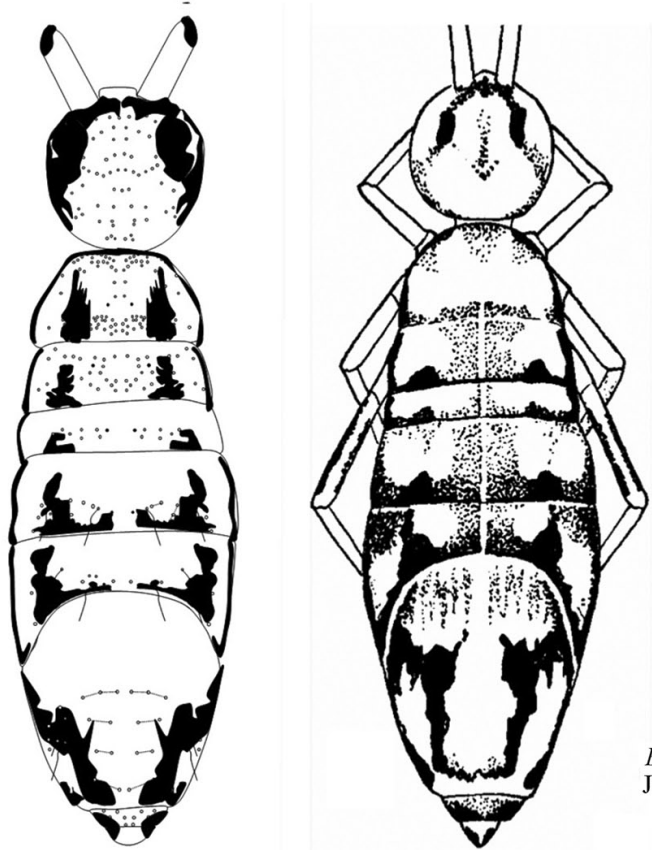

E. leonensis

Jordana \& Baquero, 2005

Fig. 3: Pigmentmuster von je 2 Exemplaren von Entomobrya nivalis und E. leonensis. Nach Jordana \& Baquero (2005). 
Figur 3 zeigt vier Entomobryiden, bei denen man - nach heutiger Kenntnis - nicht sagen kann, ob sie ein arttypisches oder ein variables Muster tragen. Die Zuordnung dieser vier Zeichnungsmuster zu den angegebenen Arten haben Jordana \& BaQuero (2005) erstmals grundsätzlich auf eine chaetotaktische Basis gestellt. Das ist hier deshalb so schwierig, weil bei diesen Gruppen die Plurichaetose, d.h. die Entwicklung eines übermäßig dichten Haarkleides, das Aufstellen chaetotaktischer Formeln über den ganzen Körper unmöglich macht. Zur Überwindung dieser Probleme schlagen die Autoren vor, mit lokalisierbaren „hot spots“ zu arbeiten; d. h. nur sorgfältig ausgewählte, überschaubare Stichproben der Chaetotaxie zu prüfen (Fig. 4). Diese Methodik wird - so steht zu hoffen - auch die Entomobryiden in praktikabler Weise der modernen Taxonomie zugänglich machen. Prof. Jordana hat zugesagt, die Entomobryiden in den „Synopses“ zu bearbeiten.

Komplexarten: Entsprechend des noch niedrigen Standes der Taxonomie vereinigen nicht wenige Artbeschreibungen eine unbekannte Zahl real existierender Arten („Komplexarten“). Die Verwirrung erhöht sich, wenn „Wiederbeschreibungen“ ohne Kontrolle des Holotypus vorgenommen wurden. Steht der Holotypus noch zur Verfügung, ist oft eine Aufklärung auf der Basis der modernen Taxonomie möglich (z. B. Potapov \& Dunger 1999). Weil die Freude der Neubeschreibung näher liegt als die Mühen der basalen Taxonomie, wird die Kontrolle der fraglichen Taxa noch lange Zeit in Anspruch nehmen. Einen ersten Schritt zur Beseitigung der Komplexarten gehen die Autoren der Synopses, indem sie diese als solche kennzeichnen und zur weiteren Prüfung anregen.

Ein bereits aufgeklärtes Beispiel ist die von BöRnER (1901) als Mesaphorura krausbaueri beschriebene Komplexart, die noch von Gisin (1955) unter Tullbergia krausbaueri als Charakterart guter Weinbergböden und von GHilarov (1965) generell als Indikator fruchtbarer Böden bezeichnet wurde. Figur 5 zeigt, was seit 1965 hiermit geschah. Die Entdeckung von Unterschieden an zwei Sinnesorganen, am dritten Antennenglied und postantennal gelegen, veranlasste Rusek $(1971$, 1974) und weitere Autoren zur Beschreibung neuer Gattungen. Unterschiede in Zahl und Position von Körperborsten und Pseudocellen wurden zur Begründung neuer Arten verwendet. So entstanden in 30 Jahren aus der in jedem Boden massenhaft vorhandenen "Tullbergia krausbaueri“ 12 Gattungen mit 65 Arten, die vorwiegend parthenogenetische Morphospecies darstellen. Der hohen Bedeutung wegen haben Zimdars \& Dunger (2000) Populationen von vier ausgewählten Mesaphorura-Arten aus fünf Provenienzen einem komplexen Test unterzogen. Geprüft wurden die Konstanz der morphologischen Merkmale in Zuchten unter verschiedenen Temperaturbedingungen, das ökologische Verhalten in diversen Freilandsuntersuchungen sowie die Ergebnisse von Enzymelektrophoresen und DNAUntersuchungen (RAPD-PCR; Restriktionsanalyse). Geplante Kreuzungsexperimente waren wegen der Parthenogenese der verfügbaren Populationen nicht möglich. Das Ergebnis lautet kurz gefasst: der Artstatus kann nicht bezweifelt werden. Dieses Resultat bestätigt, dass das hoch differenzierte ökologische Verhalten der Mesaphorura-Arten (Zimdars \& Dunger 1994) zu Recht für Indikationen herangezogen werden kann. Für die praktische Arbeit bedeutet dies aber auch, dass nunmehr gute Ökologen ein Mehrfaches an Zeit am Mikroskop aufwenden müssen, um die „Tullbergien“ artgerecht zu erfassen.

Genetische Differenzierung: Molekularchemische Daten haben heute hohe Bedeutung für phylogenetische Ableitungen oder zur Aufklärung von sibling species wie auch der Bewertung von Farbvarianten (FraTI et al. 1989). Als hilfreich für die Taxonomie der Collembolen bewähren sich molekularchemische Untersuchungen besonders dann, wenn es gelingt, den genetischen Differenzen morphologisch erkennbare Merkmale zuzuordnen. 

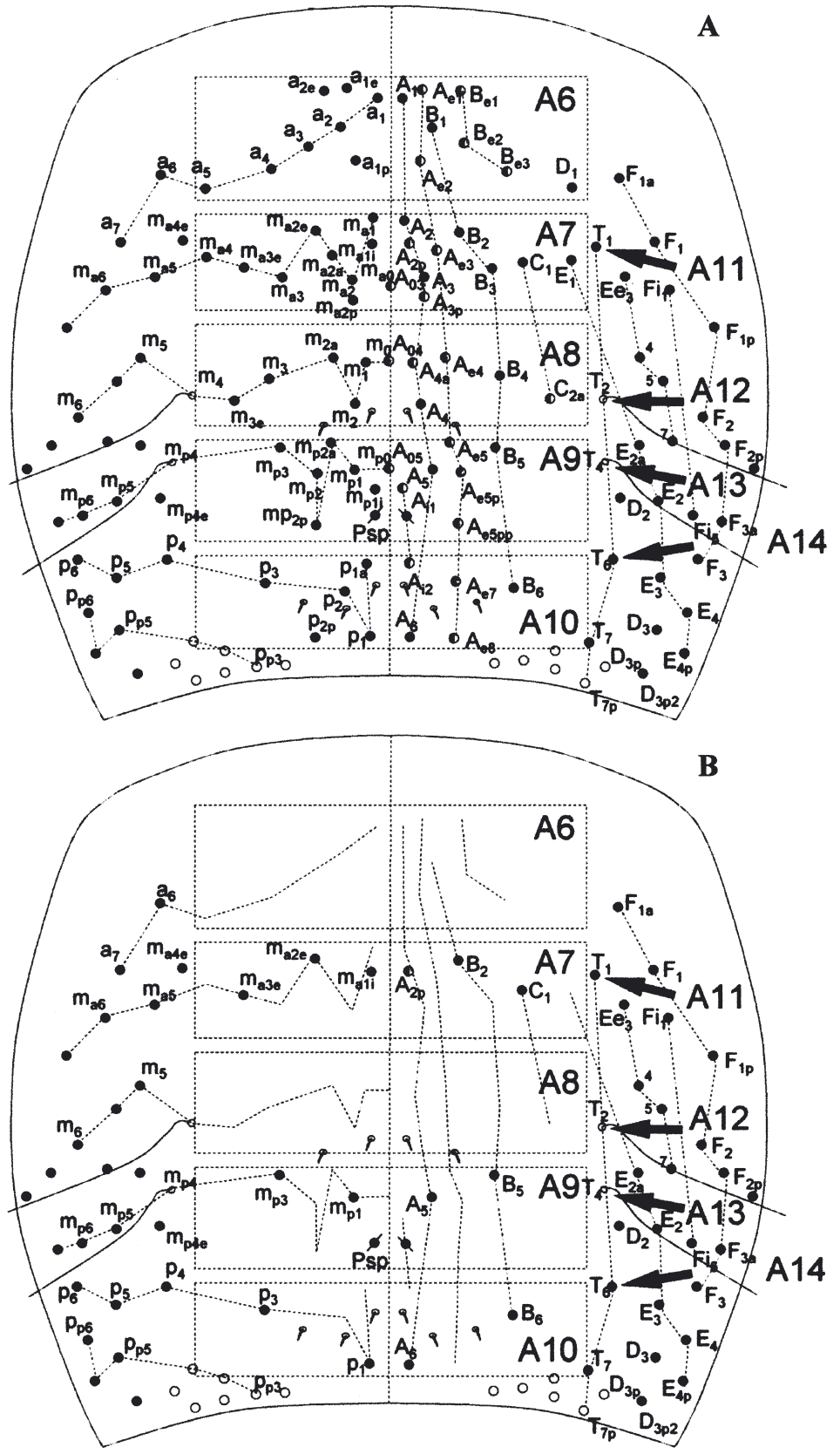

Fig. 4: Beispiele der „selektiven Prüfung“ der Chaetotaxie von Entomobrya: A: Generalisierte Chaetotaxie des 4. Abdominaltergits mit Testfeldern (A6 - A10); B: konkrete Chaetotaxie mit arttypischer Besetzung der Testfelder bei Entomobrya nivalis (A $11-$ A14 = Trichobothrien) (s. Text). Nach JordANA \& BaQuero (2005). 


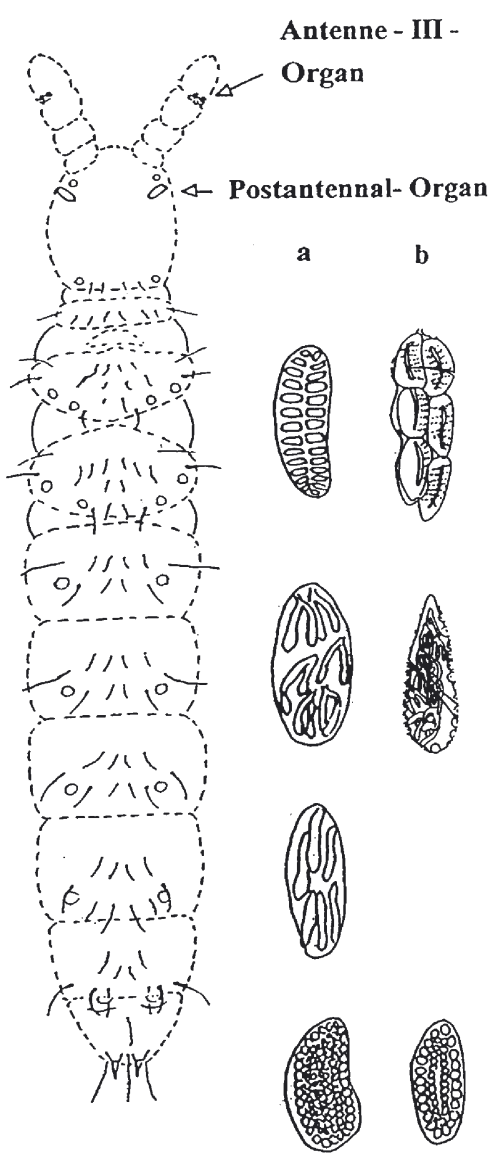

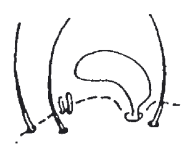

I

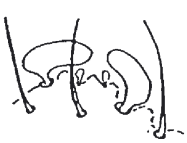

u

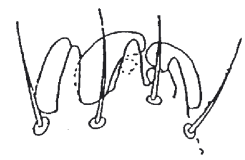

III

I a Doutnacia Rusek, 19742 species

I a Najtiaphorura Weiner \& Thibaud, 19912 species

II a Mesaphorura Börner, 190137 species

II a Pongeiella Rusek, 19912 species

III b Fissuraphorura Rusek, 19912 species

I a Jevania Rusek, 19782 species

II a Wankeliella Rusek, 19754 species

U b Marcuzziella Rusek, 19751 species

A a Karlstejnia Rusek, $1974 \quad 5$ species

I a Scaphaphorura Petersen, 19652 species

II a Multivesicula Rusek, 19825 species

II b Granuliphorura Rusek, 19.761 species

\section{Gegenwärtig 12 Genera mit 65 Species}

Fig. 5: Holarktische Taxa der „Tullbergia krausbaueri-Gruppe“, mit Darstellung der Ausprägung der „Antenne-III-Organe“ (römische Zahlen) und der „Postantennal-Organe“ (Kleinbuchstaben). Aus Dunger (1991), verändert.

Ein typisches Beispiel bietet der morphologisch sehr einheitliche „Riesencollembole“ Tetrodontophora bielanensis. Sein disjunktes Verbreitungsgebiet umfasst im Nordosten die Sudeten und Karpaten mit weitem Vordringen längs der Flüsse nach Norden, im Südwesten dagegen die Ostalpen mit Slowenien und Kroatien. Fanciulli et al. (1991) stellten eine hohe genetische Distanz zwischen den nordöstlichen (Polen, Sudeten) und südwestlichen (Italien, Ostalpen) Populationen mittels der Isozym-Variabilität fest. Die morphologische Widerspiegelung dieser Unterschiede und die taxonomischen Folgerungen lassen allerdings seither auf sich warten. 
Tab. 2: Publikationsplan der „Synopses on Palaearctic Collembola“ nach dem Stand von Mitte 2005.

\section{Synopses on Palaearctic Collembola (updated edition plan 2006)}

Edition: Wolfram Dunger; English language: David Russell, Steve Hopkin

Published in the „Abhandlungen und Berichte des Naturkundemuseums Görlitz”, editor-in-chief Prof. Dr. Willi Xylander

In preparation:

I General Introduction: Wolfram Dunger

(with a key to the supraspecific taxa of Collembola of the Palaearctis)

Modern studies of Collembolan phylogeny: Francesco Frati; acc. 2003

General index for Vol.s I - VIII

Appeared:

I (outdated) Tullbergiinae: Bettina Zimdars, Wolfram Dunger (71 pp)

II Symphypleona (incl. Neelipleona): Gerhard Bretfeld (318 pp)

III Isotomidae: Mikhail Potapov (603 pp)

IV Hypogastruridae: Jean-Marc Thibaud, Hans-Jürgen Schulz,

In preparation:

V Neanuroidea: Louis Deharveng; acc. 1999

Pars 1: Morulinae + Neanuridae

Pars 2: Remaining Neanuridae + Brachystomellidae

VI Onychiuroidea

Onychiuridae: Romuald Pomorski; acc. 2000

Tullbergiidae: Wolfram Dunger; acc. 2003

VII Entomobryoidea

Cyphoderidae: Walter Hüther; acc. 1999

Entomobryidae

Entomobryinae: Rafael Jordana; acc. 2003

Lepidocyrtinae: Walter Hüther; acc. 1999

Orchesellinae: Mikhail Potapov; acc. 2002

Seirinae: Walter Hüther; acc. 1999

VIII Collembola varia

Tomoceroidea

Oncopoduridae: Louis Deharveng, acc. 2001

Tomoceridae: Walther Hüther; acc. 1999

Odontellidae: Javier Arbea; acc. 2002

Paronellidae: $N N$

Collembola incertae sedis

Gulgastruridae, Isotogastruridae, Mackenziellidae, Poduridae:

Wolfram Dunger; acc. 2004 
Synopses on Palaearctic Collembola

Jeder Versuch, die Collembolen der Paläarktis darzustellen, muss die vorstehend geschilderte Situation der taxonomischen Kenntnis beachten. Um 1990 begann der Autor mit der Erarbeitung einer Übersicht über die Collembolen der Paläarktis auf zwei Ebenen: Erstens galt es, führende Spezialisten zur taxonomisch kritischen Bearbeitung der ihnen am besten bekannten Gruppe (Familien) zu gewinnen, und zweitens, mit der Bearbeitung der Tullbergiinae (heute Tullbergiidae) der Paläarktis (Zimdars \& Dunger 1994) ein Beispiel vorzugeben.

Dieser als Band I der „Synopses“ erschienene Band wird künftig durch eine englische Neubearbeitung im Rahmen des Bandes VI (Onychiuroidea) ersetzt, weil er mit dem „Makel der nicht-englischen Sprache" ebenso behaftet ist, wie das französische Manuskript des Bandes IV (Hypogastruridae), das noch vor der Drucklegung ins Englische übersetzt werden musste. Auch die Collembolenkunde unterliegt der Gleichschaltung im anglizistischen Zeitalter.

Die Planung ging von der Erwartung aus, dass das Staatliche Museum für Naturkunde Görlitz mit seiner Abteilung Bodenzoologie ein geeigneter Träger sein wird, mit Fachkompetenz, ausreichender Druckkapazität und ökonomischen (Förder-)Möglichkeiten (z. B. durch die DFG).

Es wurden 8 Bände mit je 300 - 600 Druckseiten geplant, die mit 1 - 2 Jahren Abstand erscheinen sollten. Hiernach müsste in diesem Jahr (2006) der Schlussband ausgeliefert werden - real ist 2006 die Fertigstellung von Pars 1 des Bandes V zu erwarten. Den aktuellen Editionsplan zeigt Tabelle 2.

Die bisher erschienenen Bände der Synopses beschreiben knapp die Hälfte der paläarktischen Arten der Collembola (Tab. 3). Das Resultat lässt erkennen, dass etwa $40 \%$ der Gattungen und $57 \%$ der Arten der Paläarktis erst nach 1960 beschrieben wurden, wobei die Tullbergiidae mit über $60 \%$ der Gattungen und $80 \%$ der Arten die intensivste Neubearbeitung erfuhren. Die Taxonomie der Symphypleona auf der Artenebene blieb mit nur etwa $46 \%$ der Beschreibungen nach 1960 am konservativsten. Der mit 9,2\% hohe Anteil der species inquirenda (118 Arten) zeigt, dass die Taxonomie der Collembola auch in der Paläarktis noch stark im Fluss ist.

Hinsichtlich der Artenzahlen stellt Tabelle 4 das zu erwartende Gesamtergebnis der Synopses dar. Gegenwärtig stehen noch etwa 1800 Arten zur Bearbeitung. Mit 3100 Arten scheint der heute erschließbare Artenbestand der Collembolen in der Paläarktis annähernd real eingeschätzt zu sein. Die taxonomische Kenntnis der in den Synopses noch ausstehenden Supertaxa ist nicht besser, sondern eher problematischer als die der bereits im Druck vorliegenden Taxa. Also ist der Abschluß der Synopses im Jahr 2010 nur eine Hoffnung.

\section{Nutzung der zu erwartenden Kenntnis}

Abschließend ist die Frage nach der praktischen Nutzbarkeit der „Synyopses on Palaearctic Collembola" für Nichtspezialisten, vor allem für Ökologen verschiedenster Couleur, zu stellen. Collembolen werden in eher zunehmendem Maß für bodenbiologische Untersuchungen oder als Testorganismen zur Klärung der Wirkung von agrartechnischen Praktiken und für Labortests der verschiedensten Art herangezogen. Viele der Anwender geben - wenn überhaupt - abenteuerliche Quellen dafür an, wie sie zu den benutz- 
ten Namen der Versuchsarten gekommen sind. Der Spezialist sollte sich - eine ideeller Zwang, zumal niemand an den Synopses verdient - die Frage stellen, ob er alles Mögliche getan hat, um den Zugang zur aktuellen taxonomischen Kenntnis offenzulegen und zu erleichtern.

Tab. 3: Zahlen der Gattungen und Arten der bisher im Rahmen der „Synopses on Palaearctic Collembola“ (Bände I - IV) bearbeiteten Supertaxa.

beschriebene Gattungen

\begin{tabular}{llll} 
& vor 1960 & nach 1960 & \\
Hypogastruridae & $16(66.7 \%)$ & $8(33.3 \%)$ & 24 \\
Tullbergiidae & $7(38.9 \%)$ & $11(61.1 \%)$ & 18 \\
Isotomidae & $40(62.5 \%)$ & $24(37.5 \%)$ & 64 \\
Symphypleona & $27(62.8 \%)$ & $16(37.2 \%)$ & 43 \\
Supertaxa Bde I - IV & $90(40.4 \%)$ & $59(39.6 \%)$ & 149 \\
& \multicolumn{1}{c}{ beschriebene Arten } & \\
& vor 1960 & nach 1960 & 330 \\
Hypogastruridae & $154(46.7 \%)$ & $176(53.3 \%)$ & 75 \\
Tullbergiidae & $15(20.0 \%)$ & $60(80.0 \%)$ & 615 \\
Isotomidae & $243(39.5 \%)$ & $372(60.5 \%)$ & 260 \\
Symphypleona & $139(53.5 \%)$ & $121(46.5 \%)$ & 1280 \\
Supertaxa Bde I - IV & $551(43.0 \%)$ & $729(57.0 \%)$ &
\end{tabular}

Diese Frage hat in den Diskussionen der Spezialisten-Tagung 'Taxonomy and Ecology on Palaearctic Collembola' (Dunger 2003) eine wichtige Rolle gespielt. Sie hat dazu geführt, dass besonders bei Arten, die häufig in Laborprüfungen oder in anderem ökologischen Kontext eine Rolle spielen, spezielle Informationen in die Diskussionsteile der Artbeschreibungen aufgenommen wurden. Eine besondere Crux sind und bleiben die Schlüssel, ein wichtiges Kampffeld zwischen Autor und Herausgeber.

Noch ist der einführende Band I mit dem Basalschlüssel für alle Collembolen nicht erschienen, d. h. der Nichtspezialist muss alte Schlüssel zur Feststellung der Familienzuordnung nutzen. Mit Abschluss der Serie beginnt für das Team der Synopses die Periode der updates.

Als positiver Widerhall ist der Literatur zu entnehmen, dass die Neubearbeitungen konsequent genutzt werden. Viele Ökologen werden vielleicht den Wunsch haben, diese Informationen konzentrierter und auf ihre Belange besser abgestellt zu erhalten. Das ist durch Hopkins (1997) Ökologie der Collembolen im Grunde schon erfüllt. 
Tab. 4: Nachgewiesene bzw. geschätzte Artenzahlen der Collembolen der Paläarktis.

In den „Synopses“ I - IV nachgewiesene Artenzahlen

$\begin{array}{lr}\text { Hypogastruridae } & 330 \text { Arten } \\ \text { Tullbergiidae } & 75 \text { Arten } \\ \text { Isotomidae } & 615 \text { Arten } \\ \text { Symphypleona } & 260 \text { Arten }\end{array}$

Synopses $1994-20041282$ Arten nachgewiesen

In den „Synopses“V - VIII noch zu bearbeiten:

$\begin{array}{lr}\text { Neanuroidea } & 600 \text { Arten } \\ \text { Onychiuridae } & 400 \text { Arten } \\ \text { Entomobryoidea } & 620 \text { Arten } \\ \text { Tomoceroidea } & 105 \text { Arten } \\ \text { Übrige Taxa } & 85 \text { Arten }\end{array}$

Synopses 2006-2010: 1810 Arten geschätzt

Paläarktis 2006 (50 \% geschätzt) 3100 Arten

Bei Benutzung der Synopses ergibt sich aber der große Vorteil, dass der Ökologe wahrnimmt, wie diffizil die Unterscheidung der Arten ist und wie nahe eine Fehlbestimmung und damit eine schwerwiegende Änderung der ökologischen Aussage liegt.

Eine problematische Auswirkung kann die Verbreitung der modernen Taxonomie der Collembola - hier durch die Palaearctic-Synopses - durchaus haben: Die Zeit der raschen „Bilderbuchbestimmung" vor allem großer Collembolen (nach dem Farbmuster) ist vorbei, und sie wird auch nicht durch die Einführung von „DNA bar coding“ ersetzbar sein. Alle Bearbeiter müssen mehr Zeit zur Bestimmung von Collembolen einplanen - selbst von großen epedaphischen Arten. Das wird zwar niemand bezahlen wollen, es steht aber dennoch zu hoffen, dass die Forderung nach einer auf Artenkenntnis gestützten Ökologie auch in der Bodenbiologie (Dunger 1997) hierdurch keinen Abbruch erleidet.

Die „Synopses on Palearctic Collembola“ ersetzen keine „Fauna“ im Sinne der Darstellung eines Überblickes über eine abgeschlossene Kenntnis. Eine Fauna Palaearctica der Collembola wird wohl erst in 50 Jahren möglich sein.

\section{Dank}

Herzlichen Dank möchte ich meinen Kolleginnen und Kollegen sagen, die ihr Wissen und ihre Arbeitskraft aus freiem Willen dem Gelingen dieser Synopses gewidmet haben oder noch widmen werden: Professor Javier Arbea, Navarra, Dr. Gerhard Bretfeld, Kiel, Dr. Louis Deharveng, Paris, Professor Francesco Frati, Siena, Frau Professor da Gama Assalino, Coimbra, Dr. Walter Hüther, Bochum, Professor Rafael Jordana, Pamplona, Professor Romuald Pomorski, Wroclaw, Dr. Mikhail Potapov, Moscow, Professor Jean-Marc Thibaud, Paris und Dr. Hans-Jürgen Schulz, Görlitz. Besonderen Dank schulde ich meinem Amtsnachfolger Professor Willi Xylander für die tatkräftige Förderung des Projektes und Sicherung des Druckes sowie Dr. David Russell, Görlitz und Dr. Steve Hopkin, Reading, für die fachenglische Kontrolle der Bände. 


\section{Literatur}

Bitsch, J.; Bitsch, C.; Bourgoin T. \& D’Haese, C. 2004: The phylogenetic position of basal hexapod lineagues: morphological data contradict molecular data. - Systematic Entomology 29: 433-440.

Babenko, A. B.; Chernova, N. M.; Potapov, M. B. \& Stebaeva, S. K. 1994: Collembola of Russia and adjacent countries: Family Hypogastruridae. - Moskow: Nauka, 336 pp.

Betsch, J. M. 1977: Collemboles Symphypléones de la Mongolie (Collembola). - Annales historico-naturales Musei Nationalis Hungarici, Budapest 69: 59-88.

BRETfeld, G. 1989: Chorologie und Ökologie von sieben europäischen Arten der Collembola-Symphypleona (Insecta, Entognatha). - Zoologische Jahrbücher, International Journal for Zoological Sciences, Abteilung für Systematik, Ökologie und Geographie, Jena 117: 441-489.

Bretreld, G. 1999: Symphypleona. Synopses on Palaearctic Collembola (ed. W. Dunger), Vol. II. Abhandlungen und Berichte des Naturkundemuseums, Görlitz 71 (1): 318 pp.

Christiansen, K. \& Bellinger, P. 1980/1991: The Collembola of North America, north of the Rio Grande. - Grinnell College, Grinnell, Iowa.

Cassagnau, P. 1989: Les Collemboles Neanurinae: Elements pour une synthese phylogenetique et biogeographic. $-3^{\text {rd }}$ International Seminar on Apterygota, University of Siena: 171-182.

Dallai, R.; Malatesta, E. \& Ramellini, P. 1995: Apterygota: Collembola, Protura, Microcoryphia e Zygentoma (= Thysanura s. 1.), Diplura. - In: Minelli, A. et al. (ed.s), Checklist delle specie della fauna italiana, 33, Calderini, Bologna.

Deharveng, L. 1979: Chétotaxie sensillaire et phylogenese chez les Collemboles Arthropleona. - Travaux du Laboratoire d' Écobiologie des Arthropodes Édaphiques, Toulouse 1 (3): 1-15.

Deharveng, L. 2004: Recent advances in Collembola systematics. - Pedobiologia, Jena 48: 415-433.

Deharveng L. (in prep): Neanuroidea. Synopses on Palaearctic Collembola (ed. W. Dunger), Vol. V. Abhandlungen und Berichte des Naturkundemuseums, Görlitz, in prep.

D'Haese, C. 2002: Were the first springtails semi-aquatic? A phylogenetic approach by means of $28 \mathrm{~S}$ rDNA and optimization alignment. - Proceedings of the Royal Society of London, Series B, 269: 1143-1151.

Dunger, W. 1978: Poduromorphe Collembolen (Insecta, Collembola) aus der Mongolischen Volksrepublik. I. Onychiuridae. - Abhandlungen und Berichte des Naturkundemuseums, Görlitz 52 (6): 1-20.

Dunger, W. 1982: Collembolen (Insecta, Collembola) aus der Mongolischen Volksrepublik. II. Isotomidae. - Annales historico-naturales Musei Nationalis Hungarici, Budapest 74: 35-74.

Dunger, W. 1983: Collembolen (Insecta, Collembola) aus der Mongolischen Volksrepublik. III. Poduridae, Hypogastruridae und Neanuridae. - Annales historico-naturales Musei Nationalis Hungarici, Budapest 75: 121-137.

Dunger, W. 1985: Stand und Interesse der Erforschung der Collembolenfauna (Insecta, Apterygota) der Mongolischen Volksrepublik. - Mitteilungen aus dem Zoologischen Museum in Berlin 61 (1): 3-10.

Dunger, W. 1991: Ökologische Prüfung von Morphospecies der „Tullbergia krausbaueri-Gruppe“ (Insecta: Collembola). - Mitteilungen aus dem Zoologischen Museum in Berlin 67: 131-140.

Dunger,W. 1994: Erkenntnisse zur Evolution der Bodenarthropoden und ihre Bedeutung für die Bodenzoologie heute. - Verhandlungen der Deutschen Zoologischen Gesellschaft, Stuttgart 87 (2): 205-214.

Dunger, W. 1997: Zur Lage der Speziellen Zoologie, besonders der Systematik und Autökologie von Bodentieren. - Abhandlungen und Berichte des Naturkundemuseums, Görlitz 83: 141-144.

Dunger, W. 2003: Taxonomy and Ecology on Palaearctic Collembola as reflected in the "Synopses on Palaearctic Collembola". Short minutes on the International Conference, St. Marienthal convent, May 2003. - Saxony State Museum of Natural History Görlitz, Germany: 8 pp.

Fanciulli, P. P.; Frati, F.; Dallai, R. \& Rusek, J. 1991: High genetic divergence among populations of Tetrodontophora bielanensis (Insecta, Collembola) in Europe. - Revue d'écologie et de biologie de sol, Paris 28: 165-173. 
Fjellberg, A. 1985: Arctic Collembola. I - Alaskan Collembola of the families Poduridae, Hypogastruridae, Odontellidae, Brachystomellidae and Neanuridae. - Entomologica Scandinavica, Copenhagen 21: 1-126.

FJellberG, A. 1995: The systematic position of the monotypic family Isotogastruridae (Collembola) with description of Isotogastrura coronata n. sp. from Fuerteventura, Canary Islands. - Miscelánea zoológica 17: 123-127.

Fjellberg, A. 1998: The Collembola of Fennoscandia and Denmark. Part I: Poduromorpha. - Fauna Entomologica Scandinavica Vol. 35; Brill, Leiden: 183 pp.

Frati, F.; Fanciulli, P. P. \& Dallai, R. 1989: Biochemical approach to the systematics of Collembola. - In: Dallai, R. (ed.), $3^{\text {rd }}$ International Seminar on Apterygota, Siena: 145-155.

Ghilarov, M. S. 1965: Zoologische Methoden der Bodendiagnostik. (russ.) - Moskva, Nauka: 276 pp.

Giribet, G.; Edgecombe, G. D.; Carpenter, J. M.; D’Haese, C. A. \& Wheeler, W. C. 2004: Is Ellipura monophyletic? A combined analysis of basal hexapod relationships with emphasis on the origin of inscects. - Organisms, Diversity \& Evolution 4: 319-340.

Gisin, H. 1955: Recherches sur la relation entre la faune endogée de Collemboles et les qualités agrologiques de sols viticols. - Revue Suisse de Zoologie, Genève 62: 601-648.

Gisin, H. 1960: Collembolenfauna Europas. - Museum d'Histoire Naturelle Genève: 312 pp.

Handschin, E. 1929: Urinsekten oder Apterygota (Protura, Collembola, Diplura und Thysanura)). - In: Dahl, F. (Hsg.). Tierwelt Deutschlands, Teil 16. Fischer Verlag, Jena 1929: 150 pp.

Hopkin, S. P. 1997: Biology of the Springtails (Insecta: Collembola). - Oxford University Press: 330 pp.

Jordana, R.; Arbea, J. L. \& Arino, A. H. 1990: Catalogo de Colembolos Ibericos. Base de Datos. - Publ. Biol. Univers. Navarra, Ser. Zool. 21: 1-231.

Jordana, R. \& Baquero, E. 2005: A proposal of characters for taxonomic identification of Entomobrya species (Collembola, Entomobryomorpha), with description of a new species. - Abhandlungen und Berichte des Naturkundemuseums, Görlitz 76 (2): 117-134.

Luввоск, J. 1873: Monograph of the Collembola and Thysanura. - Ray Society, London.

Martynova, E. F. 1967: Materialy po faune nogochvostok (Collembola) Srednej Azii. (russ.) - Izvestija otdelenija biologiceskich nauk. AN Tadzhikskoy SSR 3 (28): 32-46.

Martynova, E. F. 1974: New species of springtails (Collembola) of Alpine Altai. (russ.) - New and little known species of Siberia, Novosibirsk, Nauka 8: 14-21.

Martynova, E. F. 1975: Collembola of the East Pamirs. The families Onychiuridae and Hypogastruridae. - Zoologicheskij Zhurnal 54 (3): 464-470.

Martynova, E. F. 1976: Species of genus Onychiurus Gervais, 1841 (Collembola, Onychiuridae) from the North and North-East of Asia. - Nouvelle de la Faune de Sibèrie. Acad. Sci. URSS: 5-44.

Martynova, E. F. 1986: Otrjad Collembola - nogochvostki. - Opredelitel' nasekomych Dal'nego Vostoka SSSR. Nauka, Moscow: 40-83.

Martynova, E. F. \& Vtorov, P. P. 1973: Collembola of Ghissar and Tien-Shan. - Zoologicheskij Zhurnal 52 (11): 1648-1657.

Palissa, A. 1964: Apterygota - Insekten I. Teil. - Die Tierwelt Mitteleuropas (eds, P. Brohmer; P. Ehrmann \& G. Ulmer) IV. Band, Lief. 1a, Quelle \& Meyer, Leipzig: 407 pp.

Potapov, M. 2001: Isotomidae. - Synopses on Palaearctic Collembola (ed. W. Dunger), Vol. III. - Abhandlungen und Berichte des Naturkundemuseums, Görlitz 73 (2): 603 pp.

Potapov, M. \& Dunger, W. 2000: A redescription of Folsomia diplophthalma (Axelson, 1902) and two new species of the genus Folsomia from continental Asia (Insecta; Collembola). - Abhandlungen und Berichte des Naturkundemuseums, Görlitz 72 (1): 59-72.

Rusek, J. 1971: Zur Taxonomie der Tullbergia (Mesaphorura) krausbaueri BöRneR und ihrer Verwandten (Collembola). - Acta entomol. bohemoslovaca 68: 188-206.

Rusek, J. 1974: Zur Taxonomie der Tullbergiinae (Apterygota: Collembola). - Vestnik Ceskoslovenské Spolecnosti Zoologické, Praha 38: 61-70. 
Rusek, J. 2002: Taxonomy of Collembola at the beginning of the new millenium. - Pedobiologia, Jena 46: 215-224.

Salmon, J. T. 1964: An index to the Collembola. - Royal Society of New Zealand Bulletin 7: 1-651.

SтACH, J. 1947-1963: The apterygotan fauna of Poland in relation to the world fauna of this group of insects. - Acta Monogr. Musei Hist. Natur., Kraków. Family Isotomidae 1947: 488 pp.; Families Neogastruridae and Brachystomellidae: 1949: 341 pp.; Families Anuridae and Pseudachorutidae: 1949: 122 pp.; Family Bilobidae: 1951: 97 pp.; Family Onychiuridae: 1954: 219 pp.; Family Sminthuridae: 1956: 287 pp.; Tribe Orchesellini: 1960: 151 pp.; Tribe Entomobryini: 1963: 126 pp.

Thibaud, J.-M.; Schulz, H.-J. \& da Gama Assalino, M. M. 2004: Hypogastruridae. Synopses on Palaearctic Collembola (ed. W. Dunger), Vol. IV. - Abhandlungen und Berichte des Naturkundemuseums, Görlitz 75 (2): 287 pp.

Yosı, R. 1956: Monographie der Höhlencollembolen Japans. - Contributions from the biological Laboratory - Kyoto University, Kyoto 3: 1-109.

Yosir, R. 1965: On some Collembola of Japan and adjacent countries. - Contributions from the biological Laboratory - Kyoto University, Kyoto 19: 1-71.

Yosir, R. 1970: On some Collembola of Japan and adjacent countries. II. - Contributions from the biological Laboratory - Kyoto University, Kyoto 23: 1-32.

Yosı, R. 1971: Collembola of Khumbu Himal. - In: Khumbu Himal, Universitätsverlag Wagner 4: 80-130.

Yosı, R. 1977: Critical checklist of the Japanese species of Collembola. - Contributions from the biological Laboratory - Kyoto University, Kyoto 25: 141-170.

Yoshit, R. 1992: Identity of some Japanese Collembola. - Acta Zoologica Asiae Orientalis 2: 97-110.

Zimdars, B. \& Dunger, W. 1994: Tullbergiinae. Synopses on Palaearctic Collembola (ed. W. Dunger), Vol. I. - Abhandlungen und Berichte des Naturkundemuseums, Görlitz 68 (4): 71 pp.

Zimdars, B. \& Dunger, W. 2000: Different methods for the evaluation of species of the genus Mesaphorura (Collembola, Tullbergiinae). - Pedobiologia, Jena 44: 240-247.

\section{Anschrift des Verfassers:}

Prof. Dr. Wolfram Dunger

Hofeweg 15

D - 02829 Schöpstal

Deutschland

E-Mail: Dunger-Ebersbach@t-online.de

\section{Subject editor:}

H.-J. Schulz 